\title{
A Predictive Symptoms-based System using Support Vector Machines to enhanced Classification Accuracy of Malaria and Typhoid Coinfection
}

\author{
Enesi Femi Aminu ${ }^{a}$, Emmanuel Onyebuchi Ogbonnia ${ }^{b}$, Ibrahim Shehi Shehu ${ }^{c}$ \\ ${ }^{a b c}$ Federal University of Technology, Department of Computer Science, Minna, +234, Nigeria
}

\begin{abstract}
High costs of medical equipment and insufficient number of medical specialists have immensely contributed to the increment of death rate especially in rural areas of most developing countries. According to Roll Back Malaria there are 300 million acute cases of malaria per year worldwide, causing more than one million deaths. About $90 \%$ of these deaths happen in Africa, majorly in young children. Besides malaria when tested; a large number is coinfected with typhoid. Most often, symptoms of malaria and typhoid fevers do have common characteristics and clinicians do have difficulties in distinguishing them. For instance in Nigeria the existing diagnostic systems for malaria and typhoid in rural settlements are inefficient thereby making the result to be inaccurate and resulting to treatment of wrong ailments. Therefore in this paper, a predictive symptoms-based system for malaria and typhoid coinfection using Support Vector Machines (SVMs) is proposed for an improved classification results and the system is implemented using Microsoft Visual Basic 2013. Relatively high performance accuracy was achieved when tested on a reserved data set collected from a hospital. Hence the system will be of a great significant use in terms of affordable and quality health care services especially in rural settlement as an alternative and a reliable diagnostic system for the ailments.
\end{abstract}

Index Terms: Malaria, Typhoid, Support Vector Machines, Coinfection, Microsoft Visual Basic.

(C) 2016 Published by MECS Publisher. Selection and/or peer review under responsibility of the Research Association of Modern Education and Computer Science

\section{Introduction}

According to Roll Back Malaria there are 300 million acute cases of malaria per year world wide, causing more than one million deaths. About $90 \%$ of these deaths happen in Africa, majorly in young children, Edicha et al., 2014. Malaria in Nigeria, according to the Nigerian Ministry of Health (MoH), cases roughly $60 \%$ of

* Corresponding author. Tel.:

E-mail address: 
outpatient visits to health facilities; $30 \%$ of childhood deaths; $25 \%$ of deaths in children less than one year; and $11 \%$ of maternal deaths. Interestingly, Panchbhai et al., 2012 asserted in their work that malaria perhaps originated in Africa and came along with human voyage to the Mediterranean shores, India and South East Asia. Furthermore, the Federal MoH estimates roughly 132 billion Naira spent on malaria per year in the form of treatment costs, prevention, loss of man-hours, etc. With these statistics, it is obvious that health is essential for economic prosperity.

Indeed, rural areas of developing nations in Africa are major victims of limited and few accessible medical facilities and medical personnel because they are concentrated in the urban areas. These and many other challenges in the health sector necessitated computer based diagnosis systems, Oguntimilehin et al., 2014. The fundamental reason for visiting a hospital is to get proper treatment and attention without delay from the best hands. No patient wants to be a specimen for a doctor especially one that has not improved on the knowledge gained since graduation from the medical school and at the same time, patients do not like to be delayed in receiving treatment for whatever reasons. But all these aforementioned have become the order of the day in most hospitals; especially in developing countries. Patients cannot get to see the doctor at the right time and there are some patients that find it difficult communicating their health issues and the doctor in turn has no time to keep asking the probable questions Fatumo et al., 2013. Another issue that has posed a problem in medicine in the developing world is the inadequate infusion of technology to aid the activities involved in the practice. Fatumo et al., 2013.

Typhoid fever is a worldwide health problem. Its impact is difficult to measure because the clinical picture is confused with those of many other febrile infections. Furthermore, the disease is undervalued because there are no bacteriology laboratories in most areas of developing countries; Scribd, 2015. These factors are believed to make many cases going undiagnosed. On the basis of the literature and the incidence of typhoid fever recorded in control groups in large vaccine field trials with good laboratory support it has been estimated that roughly 17 million cases of typhoid fever and 600,000 associated deaths occur annually according to Scribd, 2015.

According to the work of Onyido et al., 2012, a study of co-infection of typhoid and malaria fevers was carried out in Ekwulumili Community, Nnewi South, L.G.A., Anambra State, Nigeria, between May and July, 2012. Venous blood samples were collected from apparently healthy individuals who did not show any signs and symptoms of malaria and typhoid fevers. A total of 200 subjects comprising 52 (25\%) males and 148(74\%) females were examined. Of the 200 participants sampled, 40(20\%) tested positive for malaria, $11(5.5 \%)$ tested positive for typhoid fever and 10(5.0\%) were coinfected with malaria and typhoid fevers. More females (5.41\%) than the males (3.85\%) were coinfected with typhoid and malaria fevers. Although Pradhan, 2011 asserted that the signs and symptoms (nausea, vomiting, abdominal pain, diarrhea etc) of malaria and typhoid fevers do have common characteristics and clinicians do have difficulties in distinguishing them. The researcher equally affirmed that an incorrect elucidation of rapid diagnostic tests holdup the treatment of definite infection and concluded that further research is indispensable to enhance diagnoses of coinfection and eradicate phony interpretation of malaria rapid diagnostic tests.

The cost of advanced medical equipment and services has created a gap between those who can and can't afford it. This gap has been a major challenge in the world's healthcare program. Some part of Nigeria has suffered so much from this gap created by the cost of providing advanced medical equipment and services. In almost all our rural areas there has been an increase in death rate due to absence of specialists and most of the advanced medical equipment and facilities needed. Thus there is a pressing need for an alternative means of providing medical services especially in our rural area. To this end, the research work aimed at providing alternative system by applying SVM using the Math Lab application in malaria and typhoid support diagnosis.

\section{i. $\quad$ Support Vector Machines (SVMs)}

According to Ali et al., 2011, amongst other machine learning models; SVM which was invented by Vapnik is the most popular model that executes classification more accurate and faster than most other models in many 
application domains such as bioinformatics. The SVM is a supervised learning method that produces inputoutput mapping functions from a set of labeled training data. The mapping function is either a classification function or a regression function. In case of classification, kernel functions are usually used to map training data to a high-dimensional input space in which the input data become more separable than to the original input space. Maximum-margin hyperplanes are then found on the space. The model produced depends only on some data points of the training data near the class boundaries. Wang, 2005. SVMs regarding its high accuracy and flexibility in modeling various sources of data in the area of computational biology, an aspect of bioinformatic were further stated in the work of Dash et al., 2012. The importance of SVM classifier and its applications cannot be over emphasized. This was further affirmed in the work of Jiang et al., 2011 for classification of biological data. That is; in the problem of protein classification. The experimental results of their research work show that the string-based kernel in combination with SVM classifier presents appreciably better than the traditional spectrum kernel method.

\section{Related Works}

The research work of Oguntimilehin et al., 2015identified malaria fever as a great threat to economic growth and killer of millions of people annually especially in Africa and Asia nations. Insufficient medical experts and equipment were identified as well known factors for this ugly development in curbing the menace. Hence, the sole aim of the work was reviewing some Predictive Models that are computer-driven developed to manage malaria. And examine the future needs to present computer based feasible classifiers in diagnosis and treatment of malaria cases. This justifies the importance of our research work; which is applying SVM to classifier and diagnoses malaria and typhoid fevers.

In the article publication of Yang, 2004, the researcher stated that classification and prediction of biological data forms the primary task of bioinformatics. And considering the fast increase in the repository of biological data, it is imperative to adopt computer based approaches in classification process. He further restated that among these approaches, SVM offer the best prediction performance, in that SVMs are modeled to make the best use of the margin that separate two classes so that hidden data will adequately enhanced by the trained model. As a result, protease functional site recognition, protein function prediction, protein and DNA sequence to mentioned but few are some of the areas of bioinformatics where SVMs have been broadly applied. Thus, it is also justify applying it in diagnoses of malaria and typhoid fevers which also form an aspect of bioinformatics.

Considering the work of Chayadevi and Raju, 2014; they present an approach for automatic malaria detection with fuzzy based color segmentation, fractal feature extraction and Adaptive Resonance Theory (ART) neural network classification. Based on these features, malaria parasites in an image can be identified. Identification is done using four classifiers- ART based neural network, Support Vector Machine (SVM), Neural Network based Back propagation Feed Forward (NN-BPFF) and k-Nearest Neighbour (k-NN). These classifiers automatically classify the images as malaria and non-malaria. Thus, typhoid was not considered and it is co-infection with that of malaria.

According to the work of Widodo and Wijiyanto, 2014; they proposed a system that automatically detects tropical malaria on blood smears image using SVM that involved two main steps. Support Vector Machines method used in this study are based on binary SVM one against all. Features used are statistics, Gray Level Cooccurrence Matrix (GLCM) and Gray Level Run Length Matrix (GLRLM).

In the work of Suryawanshi and Dixit, 2013; they designed a system which is capable to detect the presence of malaria parasite within the blood cell images by comparing the test image features with the training dataset and classification is done to check whether the human host is suffering from Malaria by using Euclidean Distance Classifier \& SVM. However, results show that SVM gives better accuracy of $93.33 \%$ than that of Euclidean Distance Classifier which is $80 \%$. 


\section{The Design of Predictive Symptoms-based (Classifier) System}

The scope of data collected is within one health centre which is called Mount Moriah medical and Diagnostics Centre Nnewi/Nnobi, Anambra state, Nigeria. A total of 184 data was collected which contains a patient's symptoms and the associated lab test results which were used to developed the system via SVM. These data collected are categorized as follow.

\section{i. $\quad$ Data Variable (Input and Target)}

The input variables are those symptoms that a patient observed before coming to hospital, the test results and some biodata of the patient. They include high temperature, headache, doziness/weakness, body/abdominal pain, vomiting, age, gender, widal count and MP. Table 1 summarizes the data variable transformation. It shows input set and the range of their possible values called domains. High Temperature, body/abdominal pain, headache and doziness /weakness has two possible values which can be either yes or no i.e. 0 or 1. Age takes an integer value while gender takes one for female and zero for male. Widal count and MP are the target which can take 0,1 , or 2 depending on the level of malaria or typhoid a patient has.

Table 1. Data Variable Transformation

\begin{tabular}{|c|c|c|c|c|}
\hline S/N & INPUT SET & DOMAIN 1 & DOMAIN 2 & DOMAIN 3 \\
\hline 1 & High Temperature & Yes $=1$ & No $=0$ & \\
\hline 2 & Body/ Abdominal pain & Yes $=1$ & No $=0$ & \\
\hline 3 & Headache & Yes $=1$ & No $=0$ & \\
\hline 4 & Doziness/ Weakness & Yes $=1$ & No $=0$ & \\
\hline 5 & Vomiting & Yes $=1$ & No $=0$ & \\
\hline 6 & Age & Integer & & \\
\hline 7 & Gender & Female $=1$ & Male $=0$ & High=2 \\
\hline 8 & Widal count (Target) & $\begin{array}{c}\text { Not } \\
\text { significant }=0\end{array}$ & Medium=1 & High=2 \\
\hline 9 & MP & Not seen=0 & Medium $=1$ & \\
\hline
\end{tabular}

\section{ii. Input-Output Data Transformation}

The output data are classified based on the level of typhoid and malaria of each patient. A patient with level zero (represented as 0) is classified to be a victim of no typhoid or malaria, a patient with level one (represented as 1) is classified to be a victim of medium typhoid or malaria and a patient with level three (represented as 2) is classified to be a victim of high typhoid or malaria. Tables 2 and 3 summarize typhoid and malaria inputoutput transformation respectively. 
Table 2. Target Data Transformation for Typhoid

\begin{tabular}{|c|c|c|}
\hline Highest_widal count & Extent & Class \\
\hline Less than 80 & Not significant & 0 \\
\hline Equals 80 or 160 & Medium & 1 \\
\hline Greater than 160 & High & 2 \\
\hline
\end{tabular}

Table 3. Target Data Transformation for Malaria

\begin{tabular}{|c|c|c|}
\hline MP & Extent & Class \\
\hline No + & Not seen & 0 \\
\hline+ or ++ & Medium & 1 \\
\hline+++ and above & High & 2 \\
\hline
\end{tabular}

Tables 2 and 3 show the transformation of the widal count and MP respectively into the various classes. In Table 2 when the highest widal is less than 80 it means that the patient have no typhoid and belong to class zero(0), when patient's widal count highest is 80 or 60 then it means that the patient belong to class one(1). Finally, when a patient's highest widal count is more than 160 then the patient belong to class two (2). The same applied to Table 3 just that instead of widal count we used MP.

\section{iii. SVM Model and its Tools.}

SVM uses the concept of maximum margin hyperplane in identifying the Support Vectors (SV) which it uses to make classification decisions as shown in figure 1. The following optimization equations show how SVM is implemented in most packages like MATLAB used in this paper. The weight $\boldsymbol{W}$ (vector) with the largest margin can be deduced by obtaining the solution of the following optimization problem as in the work of Yaser et al., 2012.

$$
\text { Minimize } \frac{1}{2} W^{T} W
$$

Subject to $\boldsymbol{y}_{\boldsymbol{n}}\left(\boldsymbol{W}^{\boldsymbol{T}} \boldsymbol{x}_{\boldsymbol{n}}+\boldsymbol{b}\right) \geq 1 ; \mathrm{n}=1,2 \ldots \mathrm{N}$

Again, the optimization problem in equations 1 and 2 can be transformed in Lagrange model as in equations 3 and 4.

$$
\text { Maximize } \mathrm{L}(\alpha)=\sum_{n}^{N} \alpha_{n}-\frac{1}{2} \sum_{n=1}^{N} \sum_{m=1}^{N} \alpha_{n} \alpha_{m} y_{n} y_{m} x_{n}^{T} x_{m}
$$

Subject to $\alpha_{\mathrm{n}} \geq 0$ for $\mathrm{n}=1,2 \ldots \mathrm{N}$ and $\sum_{n=1}^{N} y_{n}=\mathbf{0}$ 
The above optimisation problem in equation 3 and 4 is a quadratic programming problem and it fits convex optimization which can be solved using any quadratic programming package like MATLAB etc, Yaser et al., 2012.

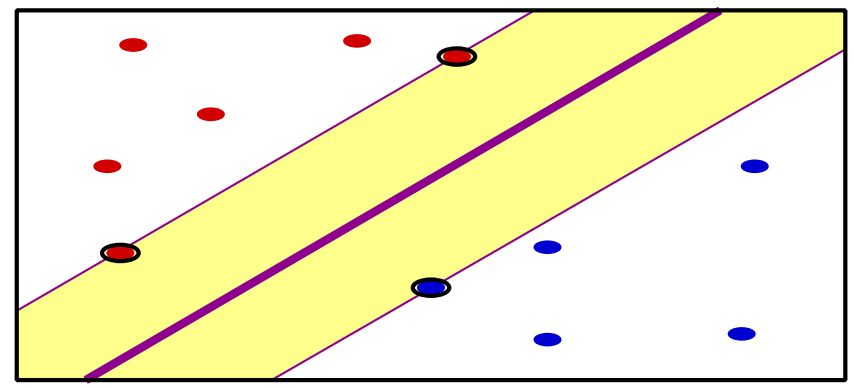

Fig.1. Support vectors; Source: cs1156x lecture slide, Yaser et al., 2012

The support vectors are all we need to solve the classification problem, Yaser, 2012. Thus the weight $\boldsymbol{W}$ and the bias $\boldsymbol{b}$ can be computed as follows:

$$
\begin{aligned}
& W=\sum_{n=s V} \alpha_{n} y_{n} x_{n} \\
& b=y-W^{T} x
\end{aligned}
$$

Where $\Sigma_{\mathrm{n}=\mathrm{sv}}$ are those points that are support vectors (that is, those points that are on the margin); $x_{n}$ corresponds to the inputs (symptoms); $y_{n}$ is the resultant class of $x_{n}$ (either not seen, medium or high as in Table 3.3 for malaria. Same applicable to typhoid); $\alpha_{n}$ is langrage multiplier. In the case of equation $6, \boldsymbol{x}$ and $\boldsymbol{y}$ is the input-output of any support vector. In order to predict new input data (symptoms) of a patient, the prediction model for the proposed system (final hypothesis $\boldsymbol{g}(\boldsymbol{x})$ ) is given as:

$$
g(x)=\operatorname{sign}\left(W^{T} x+b\right)\left\{\begin{array}{c}
\text { class } 1 \text { if } g(x)=-1 \\
\text { class } 2 \text { if } g(x)=1
\end{array}\right.
$$

The system is described to be class 1 if the model is equal to negative one, else class 2 if the model is equal to positive one. However, most of the real life data set are not linearly separable, thus a kernel $\boldsymbol{K}$ is used to map the data set into a higher feature dimension space where the data set is linearly separable. When using kernel the final hypothesis is given as in equation 8.

$$
g(x)=\operatorname{sign}\left(\sum_{\alpha_{n}>0} \alpha_{n} y_{n} K\left(x_{n}, x\right)+b\right) \quad\left\{\begin{array}{c}
\text { class } 1 \text { if } g(x)=-1 \\
\text { class } 2 \text { if } g(x)=1
\end{array}\right.
$$

Depending on the kernel been used, when a new input(x) is to be predicted, the kernel is used to computed unique value using $\mathrm{x}_{\mathrm{n}}$ and $\mathrm{x}$ which is the value used for the prediction. Hence, equation 8 is SVM typically used for binary class. However, we implored equation 9 to implement the proposed predictive symptom-based 
system for malaria and typhoid coinfection, which estimates the class likelihood of a data $(\boldsymbol{x})$. In this research work the data point is gotten when a user fills the questions on the symptom's interface of the application.

$$
p(x)=\left(\sum_{a_{n}>0} \alpha_{n} y_{n} K\left(x_{n}, x\right)+b\right)
$$

When a user fills the questions on the symptom's interface all the various classifier computes their own class likelihood. The classifiers among malaria which has the highest class likelihood estimate is selected as the predicted class, the same is also applied to typhoid classifier.

The SVM tool used in this research work is MATLAB which is one of most acknowledged tool for data analysis in science and engineering community. MATLAB being a bundle of several packages used in computation, we make use of one of the package that is designed to training SVM (fitcsvm(X,Y,Name,Value)

SVMModel $=$ fitcsvm $(\mathrm{X}, \mathrm{Y}$, Name, Value $)$ returns a support vector machine classifier with additional options specified by one or more Name, Value pair arguments. For example you can specify the type of cross validation, the cost for misclassification, or the type of score.

\section{The System Architecture}

The system architecture as shown in Fig. 2 depicts the structural view of the system and various actions that user can take in order to utilize the system. First, when a user launched the application, questions are presented on the symptom's interface for answers and then click submit button. The system will then compute class likelihood measure for all classifiers and select the classifier that has the highest value of class likelihood measure and finally the selected class is display back to the user.

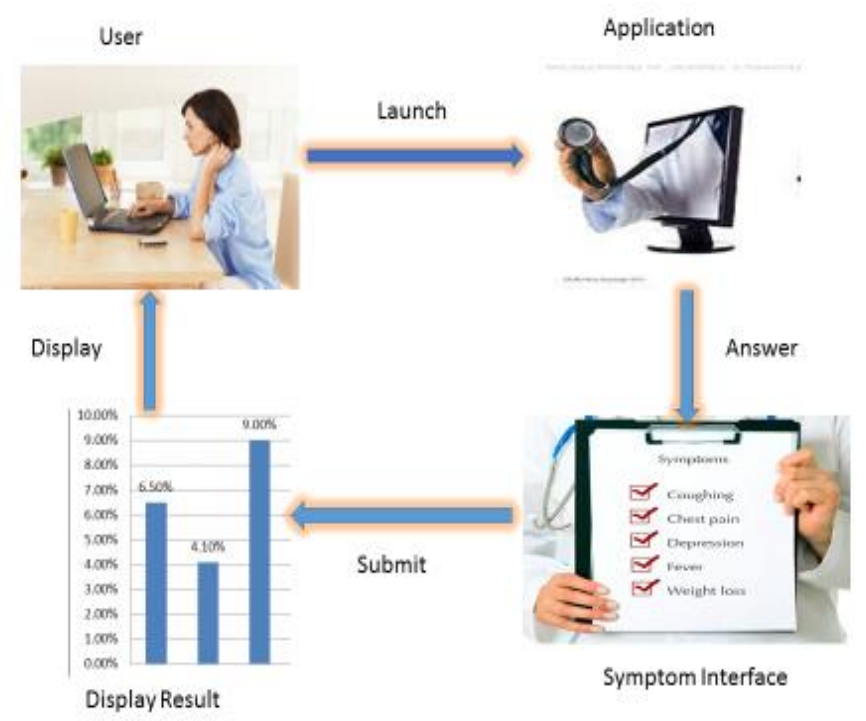

Fig.2. System Architecture 


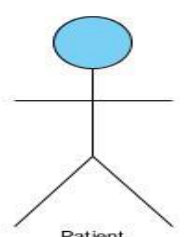

Patient

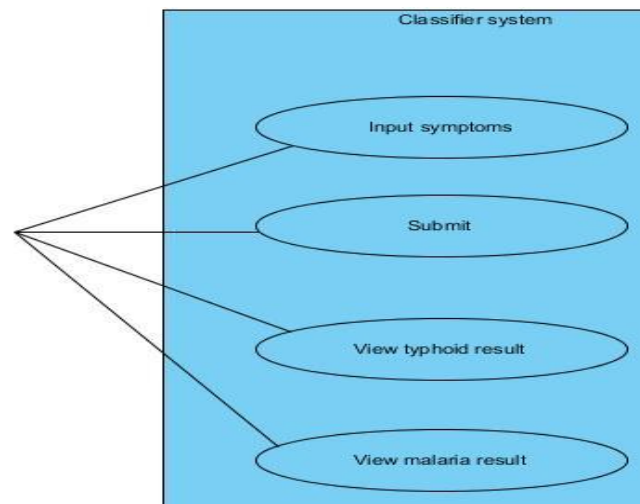

Fig.3. Use Case Diagram for the Proposed System

Table 4. Use Case Documentation

\begin{tabular}{|l|l|l|}
\hline USE CASENAME & DESCRIPTION & ACTOR INVOLVE \\
\hline Input symptoms & $\begin{array}{l}\text { The user enters his or her symptoms by answering the question } \\
\text { on the symptoms interface }\end{array}$ & Patient \\
\hline Submit & $\begin{array}{l}\text { A button on the symptoms interface where the user submits the } \\
\text { answered question }\end{array}$ & Patient \\
\hline View typhoid result & The user view the displayed typhoid result & Patient \\
\hline View malaria result & The user view the displayed malaria result & Patient \\
\hline
\end{tabular}

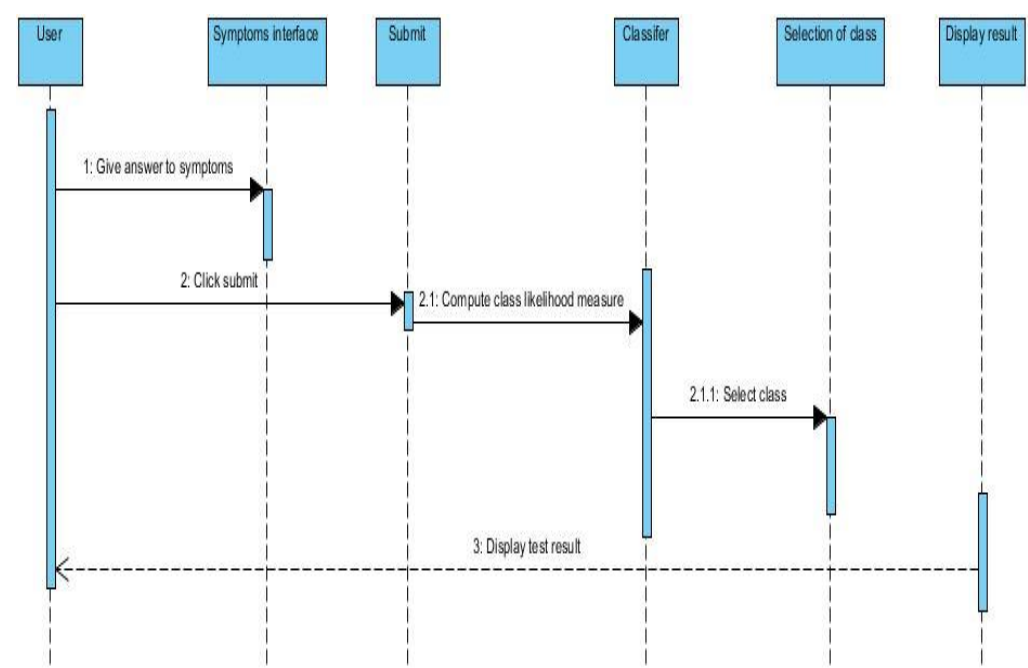

Fig.4. Sequence Diagram for the Proposed System 
Fig. 4 presents the sequence which operation follows in the system. When user launches the application, questions on the symptom's interface appeared for answer and then click submit button. The system will then compute the class likelihood measure for all the classifier and select the class with highest value of class likelihood measure. Finally, the selected class is displayed back to the user.

\section{Implementation}

The implementation of the proposed system was carried out using Microsoft excel for holding the data, Matlab for training the classifier and finally Microsoft Visual Basic 2013 for the development of the Graphical User Interface (GUI). The choice of visual basic 2013 to design the graphic user interface and code the classifiers is influenced because it is relatively simple to use and also since most of our computers runs on windows operating system, this will increase the acceptability of the proposed system since user will not find it difficult to install. This system is targeted to run on both 32 and 64bits windows operating system. The screenshots of the proposed system are shown in Figures 5 and $6 \mathrm{a}$ and $\mathrm{b}$ at design and run time respectively.

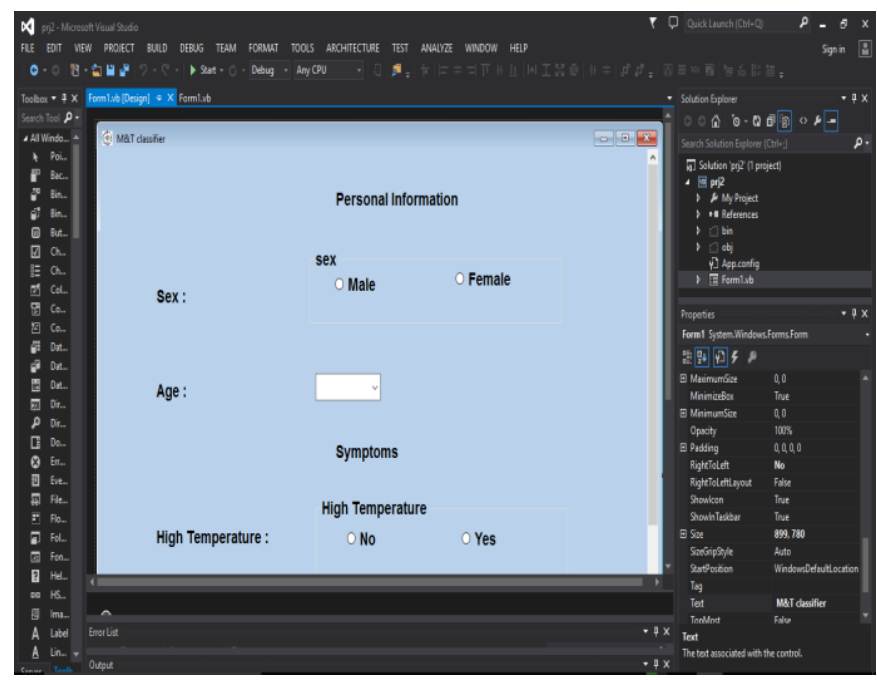

Fig.5. GUI of M \& T Predictive System at design time

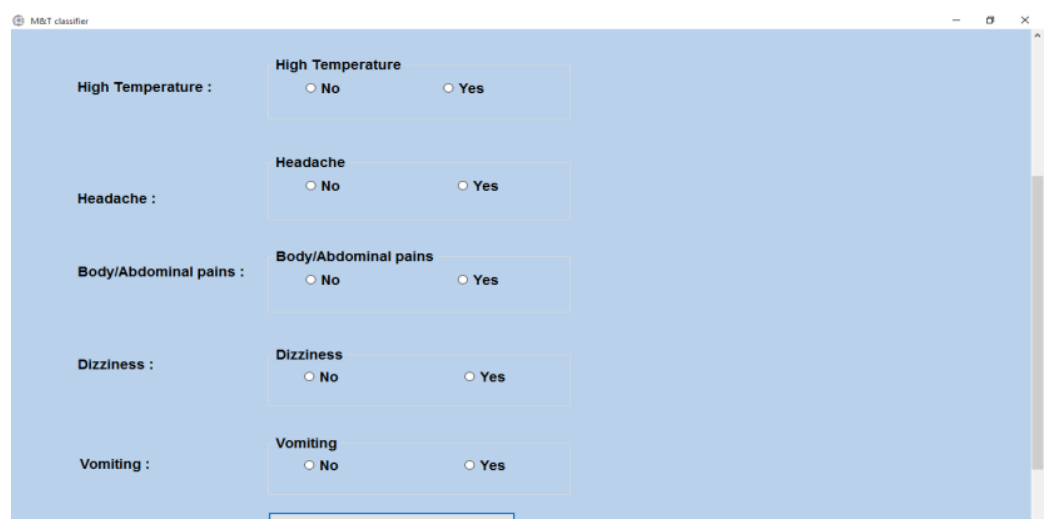

Fig.6a. M \& T Predictive System at run time 


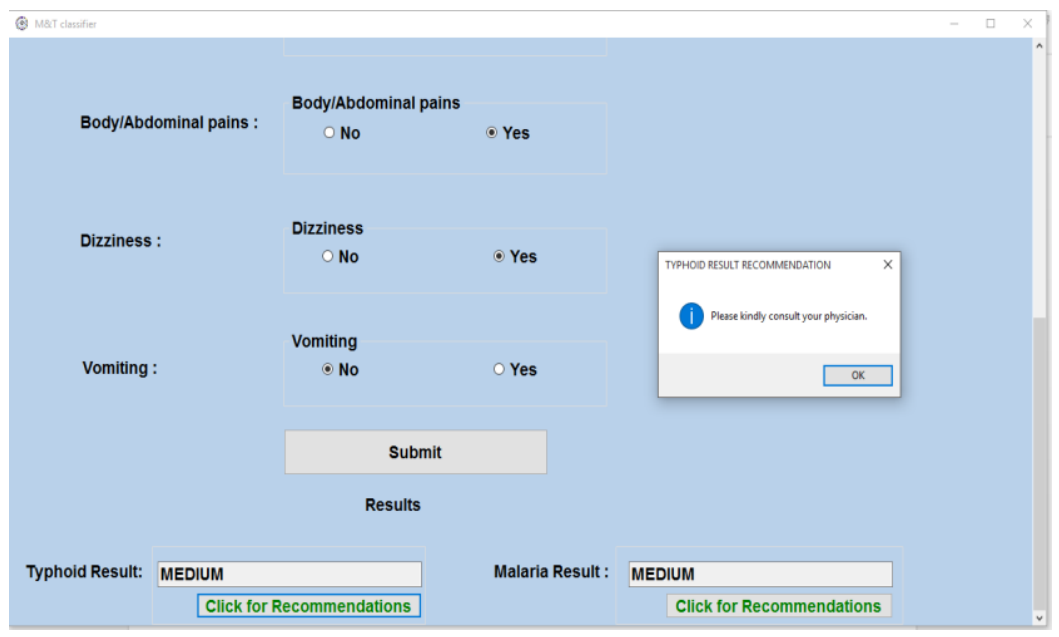

Fig.6b. M \& T Predictive System at run time

At run time, the user provides answer to the questions been displayed on the symptom's interface and click the submit button. If all the fields are properly filled, the system responds with the respective results of malaria and typhoid as shown in fig $6 \mathrm{~b}$. The user can click the recommendation button for further recommendation for advice or treatment by medical specialist. When the recommendation is clicked a message-box is displayed which shows "consult your physician with no further delay" if the result of the coinfection is high, but if the result of the coinfection is medium or not seen; the system displays " please kindly consult your physician" or "you have no problem".

\section{Results and Discussion}

Table 5 shows the result of various malaria classifiers gotten from a data set of 184 data point using OneAgainst-All. 164 data points was used for training and 20 were reserved for testing whole system. The 'Age' feature is normalized using its mean (36) and linear kernel was used in the training. The three classes that is; classes 0,1 and 2 shows $90 \%, 70 \%$ and $80 \%$ as their respective cross validation accuracy which were considered to be fair enough.

Table 5. Malaria Classifiers

\begin{tabular}{|c|c|c|c|}
\hline S/N & Class & Classifier Name & $\begin{array}{c}\text { Cross validation } \\
\text { accuracy }\end{array}$ \\
\hline 1 & 0 & SVM_M_0 & $90 \%$ \\
\hline 2 & 1 & SVM_M_1 & $70 \%$ \\
\hline 3 & 2 & SVM_M_2 & $80 \%$ \\
\hline
\end{tabular}

Table 6 equally presents the result of various typhoid classifier gotten from a data set of 184 data point, where 164 was used for training and 20 was reserved for testing. The 'Age' feature is normalised using its mean (36) and also linear kernel was used in the training. 
Table 6. Typhoid Classifiers

\begin{tabular}{|c|c|c|c|}
\hline N/S & Class & Classifier Name & Cross validation accuracy \\
\hline 1 & 0 & SVM_T_0 & $91 \%$ \\
\hline 2 & 1 & SVM_T_1 & $57 \%$ \\
\hline 3 & 2 & SVM_T_2 & $65 \%$ \\
\hline
\end{tabular}

Thus, we used linear kernel during training on a set of 164 data point and an accuracy of $90 \%, 70 \%$, and $80 \%$ were reached for classifier of various class of malaria that is class 0 , class 1 , and class 2 respectively as shown in Table 5. Also Table 6 summarises the performance of typhoid classifiers that is $91 \%$, 57\% and $65 \%$ were reached.

\section{Summary and Conclusion}

This paper presents a predictive symptom-based system for malaria and typhoid coinfection using support vector machines to improved the classification accuracy and results of the coinfection; which is aimed at providing a computer based alternative quality healthcare solution that is effective, relatively accurate and affordable especially for developing nations like Nigeria that are challenged with shortages of medical specialists and facilities. More also, the available few medical resources are situated at urban areas which is liable to make life difficult for rural dwellers. Having using MATH lab for the SVMs, the data set was held using Microsoft Excel and the proposed system was finally implemented using MS VB 2013 so as to have a working application that will assist medical personnel as well as any trained individual.

Performance evaluation was carried out using reserved 20 data point (patients). Out of the 20 data point, 16 malaria cases were classified correctly and 4 malaria was misclassified thereby making the system $80 \%$ accuracy for classifying malaria. On the other hand, 12 typhoid cases were classified correctly and 8 were misclassified thereby the system has $60 \%$ accuracy for classifying typhoid.

\section{Further Study}

In any field of studies especially in software development, it is not unusual to develop a system without expected deficiencies or limitations. These could be associated with many factors which include the proficiency level of the researcher(s) on the subject matter or time factor. Below are the deficiencies of this system.

- Inability to train with more data points and using data set that has more features as symptoms for both ailments

- The model is solely based on data set obtained from a hospital, thus having potential of streamlining the model to a particular region of the country. To avoid this, a further research can be done using data set from various regions of the country and beyond

Therefore, further work can be carried out on this proposed system based on these limitations.

\section{References}

[1] Edicha JA, Hassan SM and Ocholi J. "An Analysis of Long Lasting insecticidal Treated Nets (LLIN) in Chanchaga LGA, Niger State, Nigeria”. Confluence Journal of Environmental Studies, 9, 8-23. 2014. 
[2] Panchbhai VV, Damahe LB, Nagpure AV, and Chopkar PN. "RBCs and Parasites Segmentation from Thin Smear Blood Cell Images" I.J. Image, Graphics and Signal Processing, 2012, 10, 54-60.

[3] Oguntimilehin AO, Adetunmbi, and Olatunji KA. "A Machine Learning Based Clinical Decision Support System for Diagnosis and Treatment of Typhoid Fever" International Journal of Advanced Research in Computer Science and Software Engineering Volume 4, Issue 6, June 2014.

[4] Fatumo SA, Adetiba E and Onaolapo JO. "Implementation of xpertmaltyph: an expert system for medical diagnosis of the complications of malaria and typhoid". IOSR Journal of Computer Engineering (IOSRJCE), ISSN: 2278-8727Volume 8, Issue 5.2013.

[5] Scribd "Typhoid Fever Paper". From http://www.scribd.com/doc/267868534/Typhoid-Fever-Paper\#scribd Retrieved on 16 October 2015.

[6] Onyido AE, Ifeadi CP, Umeanato PU, Aribodor DN, Ezeanya LC, and Ugha CN. "Co-infection of malaria and typhoid fever in Ekwuhumili community Anambra State, Southeastern Nigeria." New York Sci J. 2014; 7(7): 18-27.2012.

[7] Pradhan P. "Coinfection of Typhoid and Malaria" Journal of Medical Laboratory and Diagnosis Vol. 2(3) pp. 22-26, July 2011.

[8] Ali W, Shamsuddin SM and Ismail AS. "Web Proxy Cache Content Classification based on Support Vector Machine" Journal of Artificial Intelligence 4 (1): 100 - 109, 2011.

[9] Wang L. "Support Vector Machines: theory and applications". Springer Science \&Business Media (Vol. 177) 2005.

[10] Dash S, Patra B, and Tripathy BK. "A Hybrid Data Mining Technique for Improving the Classification Accuracy of Microarray Data Set.” I.J. Information Engineering and Electronic Business, 2012, 2, 43-50

[11] Jiang H, Wai-Ki C, and Zheng Z. "Kernel Techniques in Support Vector Machines for Classification of Biological Data" I.J. Information Technology and Computer Science, 2011, 2, 1-8.

[12] Oguntimilehin A, Adetunmbi AO, and Abiola OB. "A Review of Predictive Models on Diagnosis and Treatment of Malaria Fever" IJCSMC, Vol. 4, Issue. 5, May 2015, pg.1087 - 1093

[13] Yang ZR. "Biological Applications of Support Vector Machines" Briefings in Bioinformatics. Vol 5. no 4. 328-338. December 2004.

[14] Chayadevi ML and Raju GT. "Usage of art for automatic malaria parasite identification based on fractal features". International Journal of Video\&Image Processing and Network Security IJVIPNS-IJENS Vol: 14 No:0. 2014.

[15] Widodo S and Wijiyanto. "Texture Analysis to Detect Malaria Tropica in Blood Smears Image using Support Vector Machine". International Journal of Innovative Research in Advanced Engineering (IJIRAE) ISSN: 2349-2163 Volume 1 Issue 8. 2014.

[16] Suryawanshi S and Dixit VV. "Comparative Study of Malaria Parasite Detection Using Euclidean Distance Classifier \& SVM". International Journal of Advanced Research in Computer Engineering \& Technology (IJARCET) Volume 2 Issue. 2013.

[17] Yaser SA, Malik M and Lin H. Learning from data. New York: AMLBooks. 2012.

[18] Yaser A-M. Learning from data: Introductory Machine Learning Course. Caltech. 2012.

\section{Authors' Profiles}

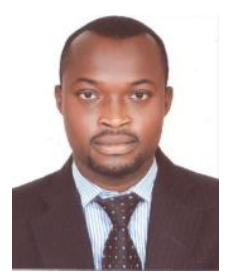

Enesi Femi Aminu is presently lectures at Computer Science Department, Federal University of Technology, Minna, Nigeria. Among courses teaching are Operating Systems, Database Design and Management, Object Oriented Programming, and Seminar. He obtained both his B.Sc and M.Sc degrees in Computer Science from University of Jos, Jos and Ahmadu Bello University, Zaria respectively. His current research interest is on ontology languages and semantic web contents. Other research interest includes Soft Computing. Also a member of 
these professional bodies: Nigeria Computer Society (NCS) and International Association of Computer Science and Information Technology (IACSIT).

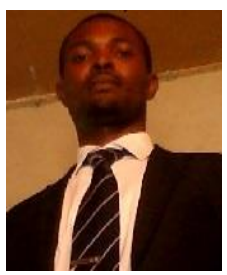

Emmanuel Oyebuchi Ogbonnia was an undergraduate student of Computer Science Department, Federal University of Technology, Minna, Nigeria; who happens to be a research project student of Mr. Enesi Femi Aminu, the first author of this paper. He graduated and awarded B.Tech in Computer Science in the year 2015. His current research interest is highly on Machine Learning Models and algorithms. He uses SVMs during his undergraduate project work.

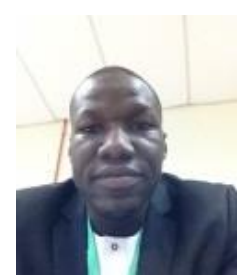

Ibrahim Shehi Shehu currently lectures in the Department of Computer Science, Federal University of Technology, Minna, Niger State, Nigeria. He obtained his M.Sc. degree in Computer Science and Entrepreneurship from the University of Nottingham in 2012. His current research motive is on the application of Decision Support Systems (DSS), Expert systems (such as Adaptive Hypermedia Systems) in education and Multi agent systems. Other research interest include; soft computing, and usability studies.

How to cite this paper: Enesi Femi Aminu, Emmanuel Onyebuchi Ogbonnia, Ibrahim Shehi Shehu,"A Predictive Symptoms-based System using Support Vector Machines to enhanced Classification Accuracy of Malaria and Typhoid Coinfection", International Journal of Mathematical Sciences and Computing(IJMSC), Vol.2, No.4, pp.54-66, 2016.DOI: 10.5815/ijmsc.2016.04.06 